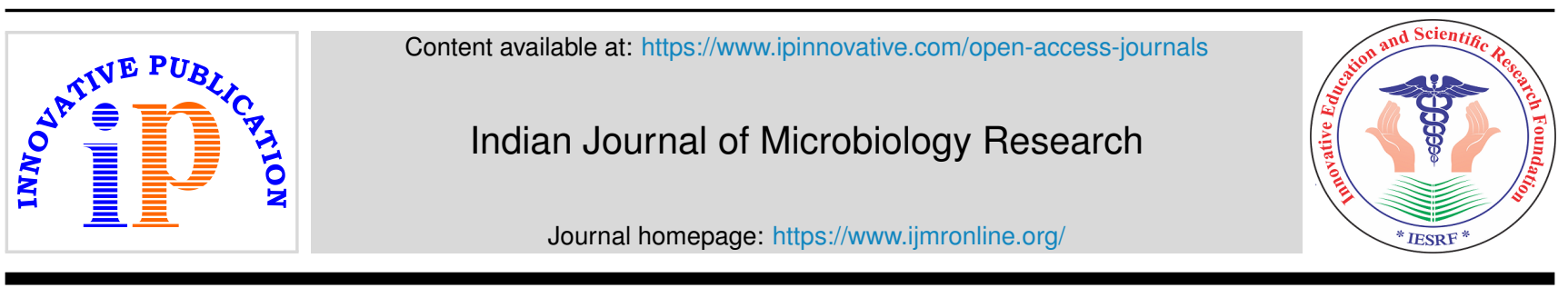

Original Research Article

\title{
A bacterial and fungal profile of sputum samples in a tertiary care hospital in Puducherry
}

\author{
Dhivya G ${ }^{1}$, R Prabha ${ }^{1} *$, Pramodhini $S^{1}$, Joshy M Easow ${ }^{1}$ \\ ${ }^{1}$ Dept. of Microbiology, Mahatma Gandhi Medical College and Research Institute, Sri Balaji Vidyapeeth, (Deemed to be \\ University), Pillaiyarkuppam, Puducherry, India
}

\section{A R T I C L E I N F O}

Article history:

Received 03-01-2021

Accepted 05-01-2021

Available online 02-04-2021

\section{Keywords:}

Gram staining

Bartlett scoring

Pathogens

\begin{abstract}
A B S T R A C T
Introduction: The microscopic examination of gram stained sputum sample aids in diagnosis of patients with lower respiratory tract infections. Gram stain plays the key role in deciding the appropriateness of the quality of the sputum sample received in the laboratory for culture. It helps to determine the represent ativeness of the sample for the site of collection intended.

Aim: This study was done to correlate gram stain findings with culture and to assess the use of Gram stain in sputum examination in diagnostic microbiology.

Materials and Methods: During 2017 (July to December) a total of 133 sputum samples were quality assessed using Bartlett's grading system. The total scoring was done and sample showing score of 1 and above were cultured and identified based on colony characteristics, gram staining morphology and biochemical reactions.

Results: One hundred and thirty-three sputum samples were collected from patients with suspected lower respiratory tract infection. Of the 133 samples, $110(79 \%)$ were accepted and $23(21 \%)$ were found to be unacceptable by Bartlett criteria. Potential pathogens were grown in 84 samples in the acceptable category. Normal respiratory flora were grown in 26 samples. Out of 84 samples, 63 samples were positive for bacterial growth and 21 showed fungal growth. Out of 63 bacterial growth, 44 were from in-patients and 19 were from out-patients. Among these bacterial isolates, 23 isolates were Pseudomonas aeruginosa followed by 16 isolates were Klebsiella pneumoniae, 10 isolates were E.coli, 6 isolates were Staphylococcus aureus, 2 isolates were Streptococcus species, 3 isolates were Proteus mirabilis and 3 isolates were Serratia marcesens.

Conclusions: All the sputum samples should be subjected to gram staining before culture to differentiate true pathogens from contaminating flora on culture.

(C) This is an open access article distributed under the terms of the Creative Commons Attribution License (https://creativecommons.org/licenses/by/4.0/) which permits unrestricted use, distribution, and reproduction in any medium, provided the original author and source are credited.
\end{abstract}

\section{Introduction}

The most practical and cost-effective diagnosis of lower respiratory tract infections rely largely on sputum culture. However, chances of contamination of the sample with saliva and the oral flora during the process of collection undermines its reliability. It also prevents futile consumption of resources and time in processing inappropriate contaminated samples that are of no benefit to the management of the patients. ${ }^{1}$ Increasing the use of

\footnotetext{
* Corresponding author.

E-mail address: philomeena.rajendran@gmail.com (R. Prabha).
}

microscopy prior to culture may increase the specificity of the sputum culture in diagnosis of respiratory infections.

Poor correlation between the culture and gram stain does not provide relevant information required concerning the aetiology of lower respiratory infection, leading to delay and indecisiveness in the management of patients. ${ }^{2}$ Identification of a pathogen by isolation from sputum culture cannot be reliably considered the etiologic pathogen in the respiratory infection unless the contamination by oropharyngeal flora is ruled out. The presence of such a contamination can be assessed by microscopy of the 
specimen and relative quantitation of the number of squamous epithelial cells in the sample.

Contaminated samples do not provide reliable diagnostic information for further management of the patients. Interpretation of sputum culture without preliminary screening for possible contamination may lead to misinterpretation and confusion in the management of patients with respiratory infection. ${ }^{3}$ The present study was designed to examine whether the clinical microbiology laboratory should play an active role in interpreting the quality of sputum specimens based on Gram stained smears, prior to inoculation into culture media.

Various scoring systems are used for screening sputum sample for acceptance of quality, each employing different cut-offs for the number of epithelial cells and neutrophils per low per field. ${ }^{4}$

A routine Gram stain is essential for all sputum samples to objective it as purulent, before inoculation in to the culture media. Use of microscopic examination of sputum samples prevents misapplication of culture in management of patients with lower respiratory tract infections. ${ }^{5}$

\section{Materials and Methods}

This Prospective study included 133 sputum samples which were received in the Microbiology department during 2017 (July to December) at Mahatma Gandhi Medical College $\&$ Research Institute, Puducherry, India. The samples were processed within $30 \mathrm{~min}$ of receipt in the laboratory. The macroscopic examination of sputum samples were done initially. From the most purulent or mucoid portion of the specimen, a smear was made and plates like 5\% sheep blood agar, chocolate agar, MacConkey agar and Saborauds Dextrose Agar were inoculated. The smear was stained with Gram's staining and was examined for the presence of polymorphs, epithelial cells, bacterial forms and fungal elements. Sputum samples were graded according to Bartletts scoring, there should be more than 25 polymorphonuclear leukocytes and less than 10 squamous epithelial cells on low-power (x 100). SEC is found only in the upper respiratory tract, so this finding suggests oropharyngeal-contamination, whereas the presence of polymorphonuclear leukocytes, suggests material derived from the site of active infection.

The total score was made by calculating the average number of epithelial cells and neutrophils in 20-30 LPFs and sample showing score of 1 and above should be considered as an acceptable sample and a score of 0 or less should be considered as inflammation or contamination (non-acceptable sample). The inoculated culture plates were incubated overnight at $37^{\circ} \mathrm{C}$ in a carbondioxide incubator. The next day, Gram's stain was done from the growth and examined.

The organisms were identified based on colony characteristics, gram staining morphology. Respiratory potential pathogens included Staphylococcus aureus, Streptococcus pyogens, Streptococcus agalactiae, Streptococcus pneumoniae, Hemophilus influenzae, Haemophilus parainfluenzae and gram negative bacilli. Normal throat flora, such as $\alpha$ - or $\gamma$-Streptococcus, CoNS, Neisseria sps, Corynebacterium sps were discarded.

Conventional biochemical (IMViC) was done to detect upto the level of species. Batch wise testing was made to check the Quality control (QC) for freshly prepared biochemicals as well as agar plates by using CLSI guidelines. For QC, recommended bacterial strains were used like Escherichia coli ATCC 25922, and ATCC 25923 Staphylococcus aureus, ATCC Candida albicans for yeast and ATCC Aspergillus niger for molds.

\subsection{Statistical analysis}

The results obtained were analyzed using MS Excel, 2010 version, with counts, percentages and pivot tables. Descriptive measures (frequencies and percentages) were used to summarize study variables.

\section{Results}

Among 133 samples, 110(92\%) sample showed more than 25 polymorphonuclear cells and less than 10 epithelial cells.

Among 44 sputum samples from in-patients, 7(8\%) were microscopy positive, $13(15 \%)$ were positive on culture and $28(33 \%)$ were both microscopy and culture positive. Among 40 sputum samples from out-patients, $5(6 \%)$ were positive on sputum microscopy, $7(8 \%)$ were positive on culture and 24(28\%) were positive on both microscopy and culture.

Among 133 patients, 43 (32\%) were aged from 40 to 60 years followed by $27(20 \%)$ patients ranges from 20 to 39 years, $55(41 \%)$ cases were aged more than 60 years and remaining $8(6 \%)$ patients are below 20 years of age. In our study population, male $79(59 \%)$ was the predominant when compared to females54 (41\%) in our study population (Table 2) 59 (11.15\%) samples were received from the OutPatient Department (OPD) and $51(88.85 \%)$ from. In-Patient Department(IPD).

Out of 84 samples, 63 samples were positive for bacterial growth and 21 showed fungal growth. Out of 63 bacterial growth, 44 were from in-patients and 19 were from outpatients. Among these bacterial isolates, 23 isolates were Pseudomonas aeruginosa followed by 16 isolates were Klebsiella pneumoniae, 10 isolates were E.coli, 6 isolates were Staphylococcus aureus, 2 isolates were Streptococcus species, 3 isolates were Proteus mirabilis and 3 isolates were Serratia marcesens.

Among 21 fungal isolates 14 isolates were Candida albicans and 6 isolates were non-albicans Candida (3Candida tropicalis, 2-Candida parapsilosis, 1-Candida glabrata), 1-Aspergillus niger. Out of the 21 fungal growth, 12 was positive in male patients and 9 from female patients. 
Table 1: Sample distribution according to Bartlett's scoring

\begin{tabular}{llc}
\hline S.No & Bartlett's scoring & No of samples \\
1 & Acceptable category & $110(92 \%)$ \\
2. & Non-acceptable category & $23(8 \%)$
\end{tabular}

Table 2:

\begin{tabular}{|c|c|c|c|}
\hline & In-patient & Out-patient & Total (110) \\
\hline Pathogens & $44(40 \%)$ & $40(36 \%)$ & $84(76 \%)$ \\
\hline Normal respiratory flora & $7(6 \%)$ & $19(17 \%)$ & $26(23 \%)$ \\
\hline Total & $51(46 \%)$ & $59(54 \%)$ & 110 \\
\hline
\end{tabular}

Table 3:

\begin{tabular}{|c|c|c|c|}
\hline & In-patient $(n=44)$ & Out-patient $(n=40)$ & Total (n=84-pathogens) \\
\hline Microscopy positive & $7(8 \%)$ & $5(6 \%)$ & $12(14 \%)$ \\
\hline Culture positive & $13(15 \%)$ & $7(8 \%)$ & $20(23 \%)$ \\
\hline Microscopy and culture positive & $28(33 \%)$ & $24(28 \%)$ & $52(62 \%)$ \\
\hline
\end{tabular}

Table 4: Age and sex wise distribution of sputum samples

\begin{tabular}{lccc}
\hline Age group & Male & Female & Total(n=133) \\
$<20$ & $5(4 \%)$ & $3(2 \%)$ & $8(6 \%)$ \\
$20-39$ & $16(12 \%)$ & $11(8 \%)$ & $27(20 \%)$ \\
$40-60$ & $30(23 \%)$ & $13(10 \%)$ & $43(32 \%)$ \\
$>60$ & $28(21 \%)$ & $27(20 \%)$ & $55(41 \%)$ \\
Total & $79(59 \%)$ & $54(41 \%)$ & 133 \\
\hline
\end{tabular}

Table 5: Distribution of bacterial isolates in sputum sample

\begin{tabular}{llccc}
\hline S.No. & Name of the isolate & $\begin{array}{c}\text { In-patient isolates } \\
(\mathbf{n = 4 4 )}\end{array}$ & $\begin{array}{c}\text { Out-patient isolates } \\
(\mathbf{n = 1 9 )}\end{array}$ & Total (n=63) \\
1. & Pseudomonas aeruginosa & $13(15 \%)$ & $10(12 \%)$ & $23(27 \%)$ \\
2. & Klebsiella pneumoniae & $12(14 \%)$ & $4(5 \%)$ & $16(19 \%)$ \\
3. & Escherichia coli & $8(10 \%)$ & $2(2 \%)$ & $10(12 \%)$ \\
4. & Staphylococcus aureus & $4(5 \%)$ & $2(2 \%)$ & $6(7 \%)$ \\
5. & Streptococcus sp & $2(2 \%)$ & 0 & $2(2 \%)$ \\
6. & Proteus mirabilis & $2(2 \%)$ & $1(1 \%)$ & $3(4 \%)$ \\
7. & Serratia marcescens & $3(4 \%)$ & 0 & $3(4 \%)$ \\
\hline
\end{tabular}

Table 6: Distribution of fungal isolates in sputum sample

\begin{tabular}{llccc}
\hline S.No. & Name of the isolate & Male $(\mathbf{n = 1 2})$ & Female $(\mathbf{n = 9})$ & Total $(\mathbf{n = 2 1})$ \\
1. & Candida albicans & $8(10 \%)$ & $6(7 \%)$ & $14(17 \%)$ \\
2. & Candida tropicalis & $2(2 \%)$ & $1(1 \%)$ & $3(1.3 \%)$ \\
3. & Candida parapsilosis & $1(1 \%)$ & $1(1 \%)$ & $2(2 \%)$ \\
4. & Candida glabrata & 0 & $1(1 \%)$ & $1(1 \%)$ \\
5. & Aspergillus niger & $1(1 \%)$ & 0 & $1(1 \%)$ \\
\hline
\end{tabular}

\section{Discussion}

In our study $92 \%$ samples were acceptable samples and $8 \%$ were non-acceptable (Table 1), but in a study by Mariraj et al. $79 \%$ were acceptable samples and $22 \%$ were non-acceptable samples and in a study done by Rana et al. $77 \%$ were acceptable samples. ${ }^{6,7}$ In contrast, Daniel Musher et al. had reported a low percentage of $31 \%$ acceptability. ${ }^{8}$ Parry et al. reported in his study that sputum Gram smear can be a guide to the etiology of pneumococcal pneumonia. ${ }^{9}$ Whereas Ewig et al. in his study reported that sputum collection can't be used for diagnosis of community acquired pneumonia and also reported that Gram stain had a low diagnostic yield as only less number of corresponding samples showed positive growth in culture. ${ }^{10}$ Among 44 sputum samples from in-patients, in our study, 7(8\%) were microscopy positive, $13(15 \%)$ were positive on culture and $28(33 \%)$ were both microscopy and culture positive. Among 40 sputum samples from out-patients, in our study, $5(6 \%)$ 
were positive on sputum microscopy, $7(8 \%)$ were positive on culture and 24(28\%) were positive on both microscopy and culture (Table 2).

In our study elderly age is more commonly affected but in a study by Bindu Nair et al. mean age of 20.9 and equal number of males and females included in the study were affected. ${ }^{11}$ In our study population, Male $79(59 \%)$ was predominantly infected when compared to females 54(41\%) which might be explained by the fact that men are more commonly involved in outdoor activities and occupation increasing the risk of infection (Table 3 ).

In this study, 7 bacterial pathogens were isolated from good quality sputum samples which includes $S$. aureus, E.coli, P.aeruginosa, K.pneumoniae, Streptococcus spp, Proteus spp, and Serratia marcessens. In our study the most commonly isolated pathogen was Pseudomonas aeruginosa but in a study done by Murdoch et al, the most commonly isolated organism was Streptococcus pneumonia. ${ }^{12}$ It may be due to the sputum samples that were collected from patients on long term antibiotic therapy or from patients with HAP. This difference can also be due to the differences in study design, type of lesion, geographical location and climatic conditions.

Total culture positivity in the present study was $100 \%$. $57 \%$ Culture positivity was reported in other studies by Jean Lloveras et al. and $79 \%$ culture positivity in studies done by Daniel Musher et al. ${ }^{13,14}$ But only 5\% of culture positivity was reported by Ravichandran et al. ${ }^{15}$ The most common pathogen causing lower respiratory tract infection as isolated was 23 isolates were Pseudomonas aeruginosa followed by 16 isolates were Klebsiella pneumoniae, 10 isolates were E.coli, 6 isolates were Staphylococcus aureus, 2 isolates were Streptococcus species, 3 isolates were Proteus mirabilis and 3 isolates were Serratia marcesens. (Table 4).

Candida species are the most common fungal pathogens in humans. Edwards reported the $8 \%-10 \%$ of all nosocomial infections are caused by Candida sps. ${ }^{16}$ In our study infection with Candida albicans is $14(17 \%)$ and nonalbicans candida 6(7\%). In a study done by Nseir et al. it was reported that, antifungal therapy significantly reduced risk of Pseudomonas aeruginosa pneumonia. ${ }^{17}$ But in a German study by Lindau et al. it was reported that patients treated with antifungal therapy had higher mortality and pneumonia rates. ${ }^{18}$ In a study by Terraneo et alno association between antifungal therapy and clinical outcome was reported. ${ }^{19}$ Harriott and Noverr reported synergistic relationship between S.aureus and C.albicans in biofilm formation. ${ }^{20}$ Delisle et al. in his study reported that hospital stay duration and mortality was more in patients who harbored Candida in their respiratory tract. ${ }^{18}$

The previous study reported that pulmonary mycosis was more common in women compared to men. ${ }^{21}$ Another study reported that more than $60 \%$ fungal culture were positive among men, ${ }^{22}$ which is similar to our study where more number of Candida were isolated from male patients (Table 5 ). Malnutrition and long term usage of antibiotics serves as an important risk factor for fungal infection as it reduces the normal flora. C.tropicalis causes infection in patients with preexisting lung disease. ${ }^{23}$ Which is similar to our study where among non-albicans Candida, C.tropicalis is reported more in number. In the, immunocompromised host $\mathrm{C}$. tropicalis is more capable of deeper tissue invasion than C.albicans. ${ }^{24}$

In a study done by Bulpa et al. on COPD, 3.6\% of cases due to A.niger were reported among patients with invasive pulmonary aspergillosis. ${ }^{25}$ In our study $1(1 \%)$ case of Aspergillus niger has been reported (Table 5).

\section{Source of Funding}

None.

\section{Conflict of Interest}

None.

\section{Acknowledgements}

I sincerely thank Dr. S. Lokesh (Prof and Head-department of General Medicine) for guididng and helping me in completing this study.

\section{References}

1. Rio-Pertuz GD, Gutiérrez JF, Triana AJ, Molinares JL, RobledoSolano AB, Meza JL, et al. Usefulness of sputum gram stain for etiologic diagnosis in community-acquired pneumonia: a systematic review and meta-analysis. BMC Infect Dis. 2019;19.

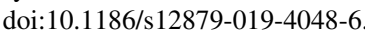

2. Miyashita N, Shimizu H, Ouchi K, Kawasaki K, Kawai Y, Obase Y. Assessment of the usefulness of sputum Gram stain and culture for diagnosis of community-acquired pneumonia requiring hospitalization. Med Sci Monit Int Med J Exp Clin Res. 2008;14(4):171-6.

3. Anevlavis S, Petroglou N, Tzavaras A, Maltezos E, Pneumatikos I, Froudarakis M, et al. A prospective study of the diagnostic utility of sputum Gram stain in pneumonia. J Infect. 2009;59(2):83-9. doi:10.1016/].jinf.2009.05.01]

4. Popova G, Boskovska K, Arnaudova-Danevska I, Smilevska-Spasova O, Jakovska T. Sputum Quality Assessment Regarding Sputum Culture for Diagnosing Lower Respiratory Tract Infections in Children. Open Access Maced J Med Sci. 2019;7(12):1926-30. doi:10.3889/oamjms.2019.551.

5. Huang WY, Lee MS, Lin LM, Liu YC. Diagnostic performance of the Sputum Gram Stain in predicting sputum culture results for critically ill pediatric patients with pneumonia. Pediatr Neonatol. 2020;61(4):420-5.

6. Mariraj J, Asangi SY, Sonth KS, Ramesh SB, Shanmugum. Sputum Gram stain assessment in relation to sputum culture for respiratory tract infections in a Tertiary Care Hospital. J Clin Diagn Res. 2011;5:1699-700.

7. Rana A, Sharma A, Pandey G. Diagnostic Value of Sputum Gram's Stain and Sputum Culture in Lower Respiratory Tract Infections in a Tertiary Care Hospital. Int J Curr Microbiol Appl Sci. 2017;6(7):4310-4. do1:10.20546/1]cmas.2017.607.448.

8. Musher DM, Montoya R, Wanahita A. Diagnostic Value of Microscopic Examination of Gram-Stained Sputum and Sputum 
Cultures in Patients with Bacteremic Pneumococcal Pneumonia. Clin Infect Dis. 2004;39(2):165-9. 101:101086/42/497.

9. Geldenhuys HD, Whitelaw A, Tameris MD, As DV, Luabeya KKA, Mahomed $\mathrm{H}$, et al. A controlled trial of sputum induction and routine collection methods for TB diagnosis in a South African community. Eur J Clin Microbiol Infect Dis. 2014;33(12):2259-66.

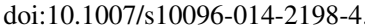

10. Ewig S, Schlochtermeier M, Göke N, Niederman MS. Applying sputum as a diagnostic tool in pneumonia: limited yield, minimal impact on treatment decisions. Chest. 2002;121(5):1486-92.

11. Nair B, Stapp J, Stapp L, Bugni L, Dalfsen JV, Burns JL. Utility of gram staining for evaluation of the quality of cystic fibrosis sputum samples. J Clin Microbiol. 2002;40(8):2791-4.

12. Khan K, Rizvi N. Yield of sputum AFB smear, gene xpert \& sputum culture of MTB in biopsy proven Pleural Tuberculosis. Eur Respir J. 2018;52. do1:10.1183/13993003.congress-2018.PA2764

13. Lloveras JJ, Shukr MI, Pinos C, Lindoulsi A, Grima P, de Luchon B. Usefulness of sputum gram stain and culture for diagnosis of pneumonia in a geriatric institution. $J I M A B$. 2010;16(3). do1:10.5272/]1mab.1632010_20-22

14. Musher DM, Montoya R, Wanahita A. Diagnostic Value of Microscopic Examination of Gram-Stained Sputum and Sputum Cultures in Patients with Bacteremic Pneumococcal Pneumonia. Clin Infect Dis. 2004;39(2):165-9. 10i:10.1086/42/497.

15. Mokkapati A. Correlation of Sputum Gram's Stain and culture in lower respiratory tract infections. IOSR J Dent Med Sci. 2013;8(1):69.

16. Singh D, Edwards L, Tal-Singer R, Rennard S. Sputum neutrophils as a biomarker in COPD: findings from the ECLIPSE study. Respir Res.

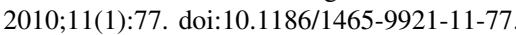

17. Nseir S, Martin-Loeches I. Ventilator-associated tracheobronchitis: where are we now? Rev Bras Ter Intensiva. 2014;26:212-4. doi: $10.5935 / 0103-507 \times 20140033$

18. Pendleton KM, Huffnagle GB, Dickson RP. The significance of Candida in the human respiratory tract: our evolving understanding. Pathog Dis. 2017;75(3):ftx029. Available from:/.

19. Terraneo S, Ferrer M, Martín-Loeches I, Esperatti M, Pasquale MD, Giunta $\mathrm{V}$, et al. Impact of Candida spp. isolation in the respiratory tract in patients with intensive care unit-acquired pneumonia. Clin Microbiol Infect. 2016;22(1):94.e1. doi:10.1016/j.cmi.2015.09.002
20. Harriott MM, Noverr MC. Candida albicans and Staphylococcus aureus Form Polymicrobial Biofilms: Effects on Antimicrobial Resistance. Antimicrob Agents Chemother. 2009;53(9):3914-22.

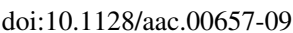

21. Astekar M, Bhatiya PS, Sowmya GV. Prevalence and characterization of opportunistic candidal infections among patients with pulmonary tuberculosis. $\quad J$ Oral Maxillofac Pathol. 2016;20(2):183.

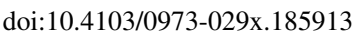

22. Bansod SD, Gupta I, Rai M. Specific detection of Aspergillus fumigatus in sputum sample of pulmonary tuberculosis patients by two-step PCR. Afr J Biotechnol. 2010;7(1).

23. Wang L, de Ángel Solá D, Mao Y, Bielecki P, Zhu Y, Sun $\mathrm{Z}$, et al. Family-based study reveals decreased abundance of sputum Granulicatella in asthmatics. Allergy. 2018;73(9):1918-21.

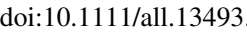

24. Najeeb T. Temporal Fascia Versus Tragal Perichondrial Graft in Myringoplasty and Tympanoplasty. Int J Otorhinolaryngol. 2019;5:15. doi:10.11648/j.1j0.20190501.14.

25. Bulpa P, Dive A, Sibille Y. Invasive pulmonary aspergillosis in patients with chronic obstructive pulmonary disease. Eur Respir J. 2007;30(4):782-800. 101:10. 103/09031036-00062206

\section{Author biography}

Dhivya G, Research Scholar

R Prabha, Assistant Professor

Pramodhini S, Professor

Joshy M Easow, Professor and Head

Cite this article: Dhivya G, Prabha R, Pramodhini S, Easow JM. A bacterial and fungal profile of sputum samples in a tertiary care hospital in Puducherry. Indian J Microbiol Res 2021;8(1):53-57. 